\title{
A new approach for an intuitionistic fuzzy Sugeno integral for decision making
}

\author{
Gabriela E. Martínez, Patricia Melin*, Oscar Castillo
}

\author{
Tijuana Institute of Technology \\ Tijuana Mexico \\ e-mail: pmelin@tectijuana.mx \\ * Corresponding author
}

Received: 20 March 2019

Revised: 29 April 2019

Accepted: 1 May 2019

\begin{abstract}
In this paper, an extension of the Sugeno integral using intuitionistic fuzzy sets is presented. The proposed method enables the calculation of the Sugeno integral for combining multiple source of information with a degree of membership and non-membership using intuitionistic fuzzy sets. The proposed method is used as aggregation operator to combine the modules output of a modular neural network for face recognition. In this paper, the focus is on aggregation operator that use measures with intuitionistic fuzzy sets, in particular the Sugeno integral. The performance of the proposed method is compared with other aggregation operators, such as the traditional Sugeno integral using the ORL database.
\end{abstract}

Keywords: Aggregation operators, Sugeno integral, Modular neural networks, Intuitionistic fuzzy sets.

2010 Mathematics Subject Classification: $03 E 72$.

\section{Introduction}

There are several applications which require handling imprecise or inaccurate data, so it is common to run into obstacles due to the uncertainty they generate. Zadeh proposed the solution in 1965 by defining fuzzy sets [26], however, Michio Sugeno completed that definition with the introduction of the fuzzy integral and fuzzy measure terms [22] as a more appropriate way to measure parameters that depend on human subjectivity, as a certain degree of uncertainty. In 1983, the concept of intuitionistic fuzzy sets was introduced by Atanassov [1], which allows having degrees of membership and degrees of non-membership. 
When the information can be mathematically formalized, combined and reduced into a single representative value, it is said that is an aggregation operator is being used. There are several aggregation operators, such as the geometric mean, the weighted average, the harmonic mean, the arithmetic mean, the ordered weighted averaging (OWA), the OWA weighting, the Sugeno integral [22] and the Choquet integral [7], each one results in the aggregation or the combination of the different sources of information that are considered as the input variables.

The Sugeno integral has been implemented successfully in several applications; in [19] interval type-2 fuzzy logic is used for module relevance estimation in Sugeno integration of modular neural networks. In [13] a comparison between Choquet and Sugeno integrals as aggregation operators for pattern recognition is presented; also in [21] the Sugeno integral is used for building the Sugeno based mean value for some specific fuzzy quantities. On the other hand, the intuitionistic fuzzy sets have had a great boom in recent years [2-4]; in [12] the concepts of interval fuzzy-valued, intuitionistic fuzzy-valued and interval intuitionistic fuzzyvalued Sugeno integrals was introduced; in [15] the Choquet and Sugeno integrals and intuitionistic fuzzy integrals as aggregation operators are presented. In [11] the theories of intuitionistic fuzzy sets and artificial neural networks are combined for intrusion detection.

In [12] it is shown that the intuitionistic fuzzy-valued Sugeno integrals are mathematically equivalent to the interval fuzzy-valued Sugeno integrals. So it is of great interest to be able to carry out the implementation of the Sugeno integral using intuitionist fuzzy logic to implement the degrees of membership and degrees of non-membership as a method of integration of responses of a modular neuronal network, applied to facial recognition, particularly in the ORL database.

Face recognition was carried out satisfactorily through the implementation of a modular network of three modules, where each one is considered as a source of information.

The rest of the paper is structured as follows: the basic concepts of the Sugeno measures and fuzzy integrals is described in Section 2, in Section 3 the extension of the Sugeno integral using intuitionistic fuzzy sets is presented. Section 4 shows the case study using the modular neural network. We explain the simulation results and the advantages of the proposed technique with benchmark face databases in Section 5, and finally Section 6 offers some conclusions of the proposed method.

\section{Sugeno measures and fuzzy integrals}

In this section, we can find the basic concepts of the Monotonic measures and the Sugeno integral.

\subsection{Monotonic measures}

A measure is considered in the area of mathematics as one of the most important concepts. We can often find in the literature that monotonous measures refer to fuzzy measures. This is somewhat confusing, because fuzzy sets are not related to the definition of these measures, due to this, in families of fuzzy sets the term "fuzzy measures" must be used for the measures (not additive or additive). 
If we have a universe of discourse $X$ and a nonempty family $C$ of subsets of $X$, a monotone measure $\mu$ on $\langle X, C\rangle$ is a function of the form $\mu: C \rightarrow[0, \infty]$. It is assumed that the universal set $X$ is finite and that $C=P(X)$. That is, normally assumed that the monotonic measures are sets of functions $\mu: P(X) \rightarrow[0,1]$.

Definition 1. On space $X$, a monotonic set measure $\mu$ is mapping $\mu: P(X) \rightarrow[0,1]$ such that the following properties hold $[10,25]$ :

1) $\mu(\phi)=0$

2) $\mu(X)=1$;

3) For all $A, B \in P(X)$, if $A \subseteq B$, then $\mu(A) \leq \mu(B)$.

\subsection{Sugeno measures}

Special types of monotonic measures are the Sugeno $\lambda$-measures and they are defined as follows $[6,17]$.

Definition 2. If we have a finite set $X=\left\{x_{1}, \ldots, x_{n}\right\}$. A discrete fuzzy measure on $X$ is a function $\mu: 2^{X} \rightarrow[0,1]$ with the following properties:

1) $\mu(\phi)=0$ and $\mu(X)=1$;

2) Given $A, B \in 2^{X}$ if $A \subset B$ then $\mu(A) \leq \mu(B)$ (monotonicity property).

The identifiers of sources of information are considered in the set $X$. For a subset $A \subseteq X, \mu(A)$ is considered to be the relevance degree of this subset of information.

Definition 3. Let $X=\left\{x_{1}, \ldots, x_{n}\right\}$ be any finite set and let $\lambda \in(-1,+\infty)$. A Sugeno $\lambda$-measure is a function $\mu$ from $2^{X}$ to $[0,1]$ with the following properties:

1) $\mu(X)=1$;

2) if $A, B \subseteq X$ with $A \cap B=\phi$, then

$$
\mu(A \cup B)=\mu(A)+\mu(B)+\lambda \mu(A) \mu(B),
$$

where $\lambda>-1$, usually the equation (1) is called the $\lambda$-rule.

The densities are interpreted as the importance of the individual information sources. If $X$ is a finite set, the fuzzy densities represented by $\mu(\{x\})$ are given for each $x \in X$, the measure of a set $A$ of information sources is interpreted as the importance of that subset of sources [17].

For each $A \subset P(X)$, the value of $\mu(A)$ can be calculated by the recurrent application of the $\lambda$-rule, and can be represented in the following form.

$$
\mu(A)=\left[\frac{\prod_{x \in A}(1+\lambda \mu(\{x\})}{\lambda}\right]
$$

For each $x \in X$, given the values of the fuzzy densities $\mu(\{x\})$, the value of $\lambda$ can be determined by using the constraint $\mu(\{x\})=1$, which applied in (2) results in (3): 


$$
\lambda+1=\prod_{i=1}^{n}\left(1+\lambda \mu\left(\left\{x_{i}\right\}\right)\right)
$$

This type of measures use the parameter $\lambda$ and once the densities are known, these can be calculated applying (3); Sugeno proved that this polynomial has a real root greater than -1 . The property (1), specifying the $n$ different densities, thereby reducing the number of free parameters from $2 n-2$ to $n$ [6], using Theorem 1, it is possible to determine the value of the $\lambda$ parameter [23].

Theorem 1. For each $x \in X$, let $\mu(\{x\})<1$ and $\mu(\{x\})>0$ for at least two elements of $X$. Then, it is possible to determine a unique parameter $\lambda$ using (3) as follows:

- If $\Sigma_{x \in X} \mu(\{x\})<1$, then $\lambda$ is a unique root in the interval $(0, \infty)$.

- If $\Sigma_{x \in X} \mu(\{x\})=0$, then $\lambda=0$; that is the unique root of the equation.

- If $\Sigma_{x \in X} \mu(\{x\})>1$, then $\lambda$ is a unique root in the interval $(-1,0)$.

Considering Theorem 1, three situations should be distinguished:

- If $\Sigma_{x \in X} \mu(\{x\})<1$, then $\mu$ qualifies as a lower probability, $\lambda>0$.

- If $\Sigma_{x \in X} \mu(\{x\})=1$, then $\mu$ is a classical probability, $\lambda=0$.

- If $\Sigma_{x \in X} \mu(\{x\})>1$, then $\mu$ qualifies as an upper probability, $\lambda<0$.

When $\mu$ is a $\lambda$-fuzzy measure, the values of $\mu\left(A_{i}\right)$ can be computed by means of (2), or recursively, reordering of the sets $X$ and $\mu(\{x\})$, with respect to the values of the elements of set $X[24]$.

\subsection{Sugeno integrals}

Sugeno develops the concept of fuzzy integral as nonlinear functions defined with respect to fuzzy measures as $\lambda$-fuzzy measure using the concept of fuzzy measures; we can interpret the fuzzy integral as finding the maximum degree of similarity between the target and the expected value as shown in (4). The Sugeno integral generalizes "max-min" operators.

$$
\text { Sugeno }_{\mu}\left(x_{1}, x_{2}, \ldots, x_{n}\right)=\max _{i=1, n}(\min (f(x \sigma(i)), \mu(A \sigma(i))))
$$

with $A_{0}=0$, where $x_{\sigma(i)}$ indicates the indices that must be permuted as shown in (5), and where $A_{\sigma(i)}=\left\{A_{\sigma(i)}, \ldots, A_{\sigma(n)}\right\}$.

$$
0 \leq f(x \sigma(1)) \leq f(x \sigma(2)) \leq \ldots \leq f(x \sigma(n)) \leq 1
$$

The Sugeno integral can be applied to solve several problems, which consider a finite set of $n$ elements $X=\left\{x_{1}, \ldots, x_{n}\right\}$. 


\section{Extension of the Sugeno integral with intuitionistic fuzzy sets}

In this section, we can find the basic concepts of the Intuitionistic fuzzy set as well as the proposed method by using the Sugeno integral

\subsection{Intuitionistic fuzzy set}

Let a set $E$ be fixed and let $A \subset E$ be a fixed sets. Intuitionistic fuzzy sets $A *$ is defined as (6)

$$
A^{*}=\left\{\left\langle x, \mu_{A}(x), v_{A}(x)\right\rangle \mid x \in E\right\}
$$

where function $\mu_{\mathrm{A}}: \mathrm{I} \rightarrow[0,1]$ and $v_{\mathrm{A}}: E \rightarrow[0,1]$ define the degree of membership and the degree of non-membership of the element $x \in E$ to the set $A$, respectively, and for every $x \in E$

$$
0 \leq \mu_{A}(x)+v_{A}(x) \leq 1
$$

Obviously, every ordinary fuzzy set has the form

$$
\left\{\left\langle x, \mu_{A}(x), 1-\mu_{A}(x)\right\rangle \mid x \in E\right\}
$$

if

$$
\pi_{A}(x)=1-\mu_{A}(x)-v_{A}(x)
$$

then $\pi_{A}(x)$ is the degree of non-determinacy (uncertainty/hesitancy) of the membership of element $x \in E$ to the set $A$. in the case of ordinary fuzzy sets, $\pi_{A}(x)=0$ for every $x \in E$, [5].

\subsection{Sugeno integral with intuitionistic fuzzy set}

Using the concept of Intuitionistic fuzzy sets is possible to extend the Sugeno integral (4) by the use of degrees of membership and degrees of non-membership, obtaining (10)

$$
\operatorname{IFSU}\left(\mu_{A}, v_{A}\right)\left(x_{1}, x_{2}, \ldots, x_{n}\right)=\left(\max _{i=1, \ldots, n}\left(\left(\min f\left(X_{\sigma_{i}}\right), \mu_{A}\left(A_{\sigma_{i}}\right)\right)\right), \max _{i=1, \ldots, n}\left(\left(\min f\left(X_{\sigma_{i}}\right), v_{A}\left(A_{\sigma_{i}}\right)\right)\right)\right)
$$

Any problem, in which there exists a finite set of $n$ elements $X=\left\{x_{1}, \ldots, x_{n}\right\}$, can be solved by applying the Sugeno integral, and the intuitionistic fuzzy Sugeno integral.

\subsection{Intuitionistic fuzzy Sugeno integral using $\pi_{A}=0.4$}

If we have that $x=\{0.9,0.6,0.3\}$ represents the information sources and associated to each entry a fuzzy density or membership value as $\mu_{i}=\{0.3,0.4,0.1\}$, we calculate $\lambda=1$.

The calculated fuzzy measures are $\mu_{A}\left(A_{\sigma(i)}\right)=\{0.3,0.82,1\}$. Using the intuitionistic fuzzy densities or not membership values as $v_{A}\left(\mu_{i}\right)=\{0.3,0.2,0.5\}$, it was calculated a $\lambda=0$ and the intuitionistic fuzzy measures calculated are $v_{A}\left(A_{\sigma(i)}\right)=\{0.3,0.5,1\}$. After that, using (10) we calculate the intuitionistic Sugeno integral 
$\operatorname{IFSU}\left(\mu_{A}, v_{A}\right)=(\max (\min ((0.9,0.3),(0.6,0.82),(0.3,0.1))), \max (\min ((0.9,0.3),(0.6,0.5),(0.3,0.1))))$ once the minimums have been calculated, the maxima are calculated

$$
\operatorname{IFSU}\left(\mu_{A}, v_{A}\right)=(\max (0.3,0.6,0.3), \max (0.3,0.5,0.3))
$$

we obtain the maxima of $\mu_{A}, v_{A}$

$$
\operatorname{IFSU}\left(\mu_{A}, v_{A}\right)=(0.6,0.5)
$$

and finally we calculate the mean of the obtained interval, however, is possible determine other way for extract a representative value of the interval obtained.

$$
\overline{\operatorname{IFSU}\left(\mu_{A}, v_{A}\right)}=0.55 \text {. }
$$

The information from different sources can be integrated with the proposed operator.

\section{Sugeno integral with intuitionistic fuzzy sets in a modular neural network}

A neural network is a computational attempt to simulate the behavior observed in human brain. The proposed method was used for the integration of the modules of a modular neural network (MNN), using a benchmark database.

\subsection{The ORL database}

The ORL contains images of the faces of 40 people with ten samples per person giving 400 images. Each image of the ORL database has a size of $112 \times 92$, in .png format [8].

In Figure 1a), we can appreciate some original images of faces and in Figure 1b) images are shown to which a preprocessing with the morphological gradient edge detector type-1 (MGT1) is applied [18].
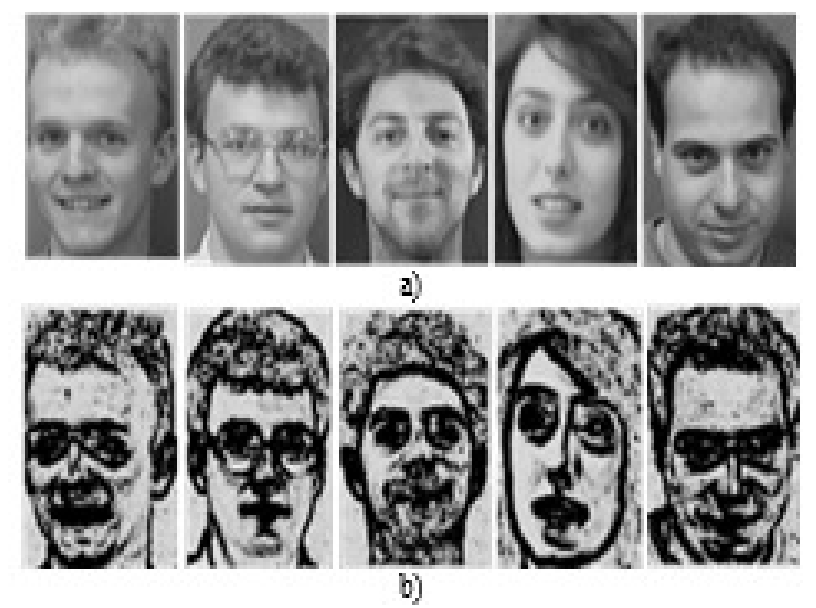

Figure 1. Images of the ORL database 


\subsection{The modular neural network}

For this work, we define a MNN of three modules using as integration of responses the Sugeno integral with intuitionistic fuzzy sets. For the MNN, we used the $80 \%$ of the images for training and $20 \%$ per testing.

Each image of the ORL database was pre-processed to improve the performance of the MNN. After that, each of the images was divided into three horizontal sections each of which serves as input to the modules of the MNN. In Figure 2, we can appreciate a scheme of the proposed architecture of the MNN.

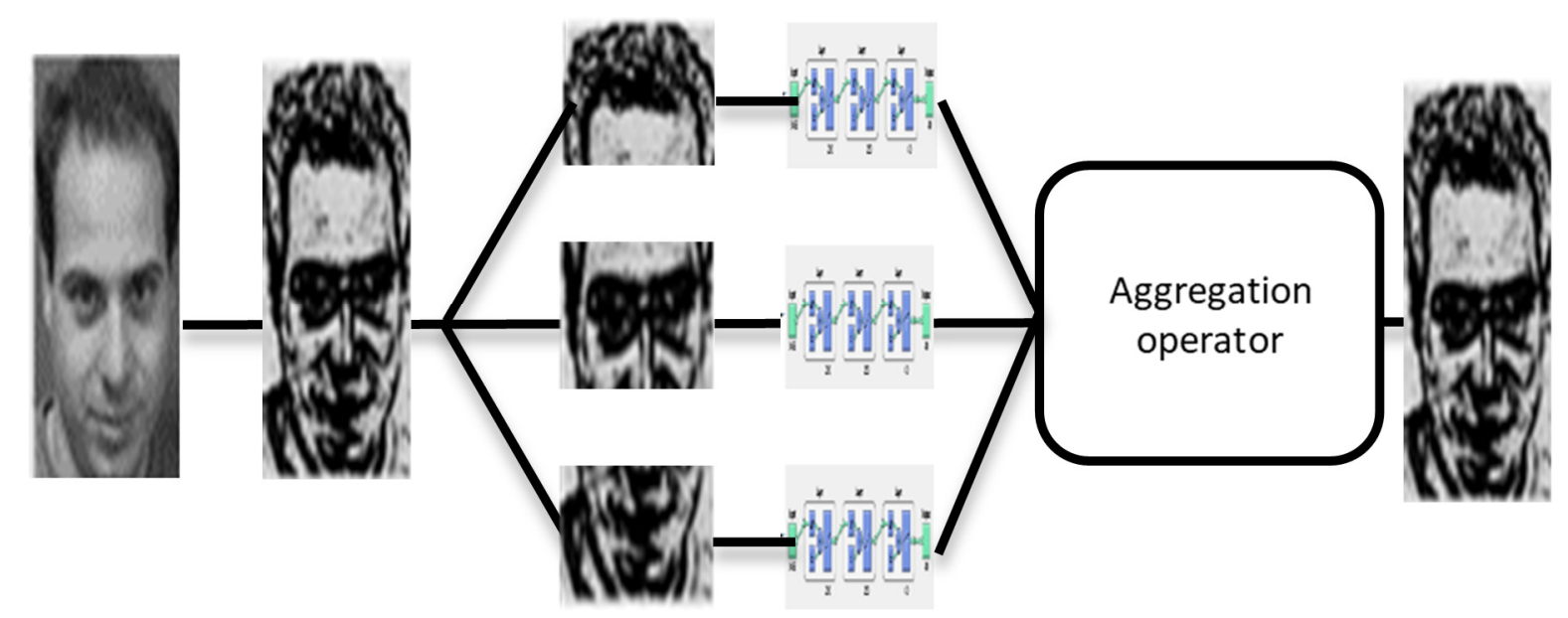

Figure 2. The modular neural network architecture

\subsection{Training parameters}

We used three modules for the training of the MNN and each one has two hidden layers with [200 200] per layer, the error goal was of 0.0001 and the number of epochs was of 500. The parameters used for the training are the gradient descendent with momentum and adaptive learning rate back-propagation (Traingdx) as training method.

With the implementation of this integral, each information source has an associated fuzzy density. The values $0.1,0.5$ and 0.9 was arbitrary chosen and some permutations were made with these values to obtain different simulations.

Using the intuitionistic fuzzy Sugeno integral is possible to determine the final decision by the combination of the simulation vectors of the outputs of the modules trained into a simple vector.

\section{Simulation results}

This section provides a comparison of the recognition rate achieved by the MNN for face recognition system were the intuitionistic Sugeno integral was applied.

In the experimental results, the MGT1 was analyzed and additional to this, the experiments were validated without using any edge detector. The tests were performed using the ORL database. In the first test, the combination of the modules output of the MNN for face 
recognition was performance without using and using the MGT1 edge detector, and applying the Sugeno integral as integration of the modules of the MNN.

The mean rate, standard deviation and the max values achieved by the system, are shown in Table 1, where we can appreciate that the better result were obtained when the MGT1 was applied with a mean rate of 89.45, a standard deviation of 0.0658 and a max rate of 97.5. Also, we can appreciated that without used the MGT1 edge detector, the results obtained show a mean rate of 2.52, a standard deviation of 0.00037 and a 3.75 as max rate.

\begin{tabular}{|l|l|l|l|l|l|l|}
\hline \multirow{2}{*}{$\begin{array}{c}\text { Test } \\
\text { number }\end{array}$} & \multicolumn{2}{|c|}{ Without edge } & \multicolumn{3}{c|}{ MGT1 } \\
\cline { 2 - 7 } & Mean & \multicolumn{1}{c|}{ Std } & \multicolumn{1}{|c|}{ Max } & Mean & \multicolumn{1}{c|}{ Std } & \multicolumn{1}{c|}{ Max } \\
\hline 1 & 0.025 & 0 & 0.025 & 0.9075 & 0.06824 & 0.975 \\
\hline 2 & 0.025 & 0 & 0.025 & 0.8925 & 0.06591 & 0.95 \\
\hline 3 & 0.025 & 0 & 0.025 & 0.89 & 0.07038 & 0.95 \\
\hline 4 & 0.025 & 0 & 0.025 & 0.8875 & 0.06847 & 0.95 \\
\hline 5 & 0.025 & 0 & 0.025 & 0.8975 & 0.06697 & 0.9625 \\
\hline 6 & 0.025 & 0 & 0.025 & 0.885 & 0.07363 & 0.95 \\
\hline 7 & 0.025 & 0 & 0.025 & 0.895 & 0.07159 & 0.9625 \\
\hline 8 & 0.025 & 0 & 0.025 & 0.9025 & 0.05687 & 0.9625 \\
\hline 9 & 0.025 & 0 & 0.025 & 0.8925 & 0.05769 & 0.9375 \\
\hline 10 & 0.0275 & 0.00559 & 0.0375 & 0.8925 & 0.05769 & 0.9375 \\
\hline 11 & 0.025 & 0 & 0.025 & 0.8925 & 0.06766 & 0.95 \\
\hline 12 & 0.025 & 0 & 0.025 & 0.9025 & 0.06337 & 0.9625 \\
\hline 13 & 0.025 & 0 & 0.025 & 0.89 & 0.07256 & 0.95 \\
\hline 14 & 0.025 & 0 & 0.025 & 0.9 & 0.06847 & 0.9625 \\
\hline 15 & 0.025 & 0 & 0.025 & 0.89 & 0.0589 & 0.9375 \\
\hline & 0.0252 & 0.00037 & 0.0375 & 0.8945 & 0.065893 & 0.975 \\
\hline
\end{tabular}

Table 1. Results using the Sugeno integral

In another test, the system was considered using the intuitionistic fuzzy Sugeno integral with a $\pi_{A}=0.1$ without using preprocessing of the MGT1 edge detector over the images of the ORL database.

In Table 2, we can appreciate that the mean rate is of 2.517 , a standard deviation of 0.00037 and a max rate of 3.75 .

In Table 3, is possible to appreciate the results obtained using the intuitionistic fuzzy Sugeno integral as aggregation operator with the preprocessing of the images with the MGT1. Using a $\pi_{A}=0.1$, we calculate a mean rate of 90.67, standard deviation of 0.96583 and max rate of 97.5 , while that with a $\pi_{A}=0.2$, the mean rate was of 89.5 , standard deviation of 0.93416 and a max rate of 95 . 


\begin{tabular}{|l|l|l|l|}
\hline $\begin{array}{c}\text { Test } \\
\text { number }\end{array}$ & Mean & \multicolumn{1}{|c|}{ Std } & \multicolumn{1}{|c|}{ Max } \\
\hline 1 & 0.025 & 0 & 0.025 \\
\hline 2 & 0.025 & 0 & 0.025 \\
\hline 3 & 0.0275 & 0.0056 & 0.0375 \\
\hline 4 & 0.025 & 0 & 0.025 \\
\hline 5 & 0.025 & 0 & 0.025 \\
\hline 6 & 0.025 & 0 & 0.025 \\
\hline 7 & 0.025 & 0 & 0.025 \\
\hline 8 & 0.025 & 0 & 0.025 \\
\hline 9 & 0.025 & 0 & 0.025 \\
\hline 10 & 0.025 & 0 & 0.025 \\
\hline 11 & 0.025 & 0 & 0.025 \\
\hline 12 & 0.025 & 0 & 0.025 \\
\hline 13 & 0.025 & 0 & 0.025 \\
\hline 14 & 0.025 & 0 & 0.025 \\
\hline 15 & 0.025 & 0 & 0.025 \\
\hline & 0.02517 & 0.000373 & 0.0375 \\
\hline
\end{tabular}

Table 2. Results using Intuitionistic fuzzy Sugeno integral with $\pi_{A}=0.1$ without edge detector

\begin{tabular}{|l|l|c|l|l|l|l|}
\hline \multirow{2}{*}{$\begin{array}{c}\text { Test } \\
\text { number }\end{array}$} & \multicolumn{3}{|c|}{$\boldsymbol{\pi}_{\boldsymbol{A}}=\mathbf{0 . 1}$} & \multicolumn{3}{c|}{$\boldsymbol{\pi}_{\boldsymbol{A}}=\mathbf{0 . 2}$} \\
\hline 1 & 0.9025 & 0.06337 & 0.9625 & \multicolumn{1}{|c|}{0.895} & \multicolumn{1}{c|}{ Std } & \multicolumn{1}{c|}{ Max } \\
\hline 2 & 0.9025 & 0.06337 & 0.9625 & 0.895 & 0.03377 & 0.95 \\
\hline 3 & 0.9025 & 0.06337 & 0.9625 & 0.9 & 0.02932 & 0.95 \\
\hline 4 & 0.9025 & 0.06337 & 0.9625 & 0.895 & 0.02739 & 0.9375 \\
\hline 5 & 0.9025 & 0.06337 & 0.9625 & 0.8925 & 0.02878 & 0.9375 \\
\hline 6 & 0.9075 & 0.06034 & 0.9625 & 0.905 & 0.01896 & 0.925 \\
\hline 7 & 0.905 & 0.05836 & 0.9625 & 0.89 & 0.03579 & 0.9375 \\
\hline 8 & 0.9025 & 0.06337 & 0.9625 & 0.895 & 0.03010 & 0.9375 \\
\hline 9 & 0.9025 & 0.06337 & 0.9625 & 0.895 & 0.03377 & 0.95 \\
\hline 10 & 0.9025 & 0.06337 & 0.9625 & 0.895 & 0.03377 & 0.95 \\
\hline 11 & 0.915 & 0.05405 & 0.9625 & 0.8925 & 0.02437 & 0.925 \\
\hline 12 & 0.915 & 0.05956 & 0.975 & 0.89 & 0.01046 & 0.9 \\
\hline 13 & 0.9125 & 0.05796 & 0.975 & 0.8825 & 0.01118 & 0.8875 \\
\hline 14 & 0.915 & 0.05956 & 0.975 & 0.9 & 0.02795 & 0.9375 \\
\hline 15 & 0.915 & 0.05956 & 0.975 & 0.9025 & 0.02404 & 0.9375 \\
\hline & 0.907 & 0.06109 & 0.975 & 0.895 & 0.02690 & 0.95 \\
\hline
\end{tabular}

Table 3. Results using Intuitionistic fuzzy Sugeno integral using MGT1 edge detector

The results obtained over the ORL database are presented in Tables 4 and 5; we can see that the recognition rate using the Intuitionistic fuzzy Sugeno integral is better than the Sugeno integral. In addition, in Table 5 we can notice that the mean rate value is better when the MGT1 edge detector is applied with a value of 90.67 using the proposed method. 
In these results we can also appreciate that the mean rate, standard deviation and max rate were better when we applied a $\pi_{A}=0.1$.

\begin{tabular}{|l|l|l|l|}
\hline & Mean & Std & Max \\
\hline Without edge & 0.0252 & 0.000373 & 0.0375 \\
\hline MGT1 & 0.8945 & 0.065893 & 0.975 \\
\hline
\end{tabular}

Table 4. Results using the Sugeno integral

\begin{tabular}{|l|l|l|l|l|}
\hline & Mean & Std & Max & $\boldsymbol{\pi}_{\boldsymbol{A}}$ \\
\hline Without edge & 0.02517 & 0.000373 & 0.0375 & 0.1 \\
\hline MGT1 & 0.907 & 0.06109 & 0.975 & 0.1 \\
\hline MGT1 & 0.895 & 0.02690 & 0.95 & 0.2 \\
\hline
\end{tabular}

Table 5. Results using Intuitionistic Sugeno integral

\section{Conclusion}

In this paper, we have presented the intuitionistic fuzzy Sugeno integral as an integration operator for a modular neural network. In Tables 4 and 5, we can appreciate that the recognition results with the proposed method are better or comparable to results produced by the Sugeno integral; however, it is necessary to perform more experimentation with different fuzzy densities. As future work, it is proposed to vary the number of information sources, as well as perform the dynamic allocation of fuzzy densities to each of the sources and perform tests with other databases. In addition, considering other applications as future work, as in [9, $14,16,20]$.

\section{References}

[1] Atanassov, K. (1983). Intuitionistic fuzzy sets, VII ITKR's Session, Sofia, June 1983 (Deposed in Central Sci.- Techn. Library of Bulg. Acad. Of Sci., 1697/84, in Bulgarian). Reprinted: Int. J. Bioautomation, 2016, 20 (S1), S1-S6.

[2] Atanassov, K. (1986). Intuitionistic fuzzy sets, Fuzzy Sets and Systems, 20 (1), 87-96.

[3] Atanassov, K. (1994). New operations defined over the intuitionistic fuzzy sets, Fuzzy Sets and Systems, 61, 137-142.

[4] Atanassov, K. (1999). Intuitionistic Fuzzy Sets: Theory and Applications, Heidelberg: Physica-Verlag.

[5] Atanassov, K., Vassilev, P., \& Tsvetkov, R. (2013). Intuitionistic Fuzzy Sets, Measures and Integrals, Bulgarian Academic Monographs Vol. 12, Sofia: "Prof. Marin Drinov" Academic Publishing House.

[6] Bezdek, J. C., Keller, J., \& Pal, N. R. (2005). Fuzzy Models and Algorithms for Pattern Recognition and Image Processing. New York: Springer-Verlag. 
[7] Choquet, G. (1953). Theory of capacities. Ann. Inst. Fourier, Grenoble, 5, 131-295.

[8] Database ORL Face. (2012, November). Cambridge University Computer Laboratory. Retrieved from: http://www.cl.cam.ac.uk/research/dtg/attarchive/facedatabase.html.

[9] González, C. I., Melin, P., Castro, J. R., Castillo, O. \& Mendoza, O. (2016). Optimization of interval type-2 fuzzy systems for image edge detection. Appl. Soft Comput. 47: 631-643.

[10] Klir, G. (2005). Uncertainty and Information. Hoboken, NJ: Wiley.

[11] Lei, Y., Liu, J., \& Yin, H. (2016). Intrusion Detection Techniques Based on Improved Intuitionistic Fuzzy Neural Networks, 2016 International Conference on Intelligent Networking and Collaborative Systems (INCoS), Ostrawva, 518-521.

[12] Liu, Y, \& Kong, Z. (2012). Interval intuitionistic fuzzy-valued Sugeno integral, Proc. of 9th International Conference on Fuzzy Systems and Knowledge Discovery, Sichuan, 8992.

[13] Martínez, G. E., Mendoza O., Castro, J. R., Melin, P., \& Castillo, O. (2014). Choquet Integral with Interval Type 2 Sugeno Measures as an Integration Method for Modular Neural Networks. Proc. of WCSC 2014, 71-86.

[14] Melin, P., \& Castillo, O. (2001). Intelligent control of complex electrochemical systems with a neuro-fuzzy-genetic approach, IEEE Transactions on Industrial Electronics, 48 (5), 951-955.

[15] Melin, P., Martinez, G. E., \& Tsvetkov, R. (2017). Choquet and Sugeno integrals and intuitionistic fuzzy integrals as aggregation operators. Proc. of $4^{\text {th }}$ International Intuitionistic Fuzzy Sets and Contemporary Mathematics Conference, Turkey, 95-99.

[16] Melin, P., Sánchez, D., \& Castillo, O. (2012). Genetic optimization of modular neural networks with fuzzy response integration for human recognition. Inf. Sci., 197, 1-19.

[17] Mendez-Vazquez, A., Gader, P., Keller, J. M., \& Chamberlin, K. (2008). Minimum classification error training for Choquet integrals with applications to landmine detection, IEEE Trans. Fuzzy Syst. 16 (1), 225-238.

[18] Mendoza, O., Melin, P., \& Licea, G. (2009). Interval type-2 fuzzy logic for edges detection in digital images. Int. J. Intell. Syst. 24(11), 1115-1133.

[19] Mendoza, O., Melin, P., \& Licea, G. (2008). Interval Type-2 Fuzzy Logic for Module Relevance Estimation in Sugeno Integration of Modular Neural Networks. Soft Computing for Hybrid Intelligent Systems, Studies in Computational Intelligence, Vol. 154, Springer, 115-127.

[20] Sánchez, D., Melin, P., \& Castillo, O. (2017). Optimization of modular granular neural networks using a firefly algorithm for human recognition. Eng. Appl. of AI 64, 172-186.

[21] Štajner-Papuga, I., Lozanov-Crvenković, Z. \& Grujić, G. (2016). On Sugeno integral based mean value for fuzzy quantities, Proc. of IEEE 14th International Symposium on Intelligent Systems and Informatics (SISY), Subotica, 155-160. 
[22] Sugeno, M. (1974). Theory of Fuzzy Integrals and Its Applications. Doctoral Thesis, Tokyo Institute of Technology.

[23] Torra, V., \& Narukawa, Y. (2007). Modeling Decisions, Information Fusion and Aggregation Operators. Heidelberg: Springer-Verlag.

[24] Verikas, A., Lipnickas, A., Malmqvist, K., Bacauskiene, M., \& Gelzinis, A. (1999). Soft combination of neural classifiers: A comparative study, Pattern Recognition Letters, 20 (4), 429-444.

[25] Yager, R. (2008). A knowledge-based approach to adversarial decision-making Int. J. Intell. Syst., 23 (1), 1-21.

[26] Zadeh, L. A. (1965). Fuzzy sets. Inform Control, 8, 338-353. 